\title{
A NOTE ON PALEY-WIENER-ZYGMUND STOCHASTIC INTEGRALS
}

\author{
CHULL PARK AND DAVID SKOUG
}

(Communicated by William D. Sudderth)

\begin{abstract}
A Fubini theorem for multiparameter Paley-Wiener-Zygmund stochastic integrals is established which unifies various stochastic integration formulas. We also obtain similar results for double stochastic integrals of Paley-Wiener-Zygmund type.
\end{abstract}

1. Introduction. Paley-Wiener-Zygmund (P. ${ }^{\times}$.Z.) stochastic integrals have been used in several recent papers, including $[4-12,17-20,28]$, concerning Feynman integration theory. In particular, P.W.Z. integrals are used in the definition of a Banach algebra $S$ of functions on Wiener space that was introduced by Cameron and Storvick [4]. In [17] Johnson showed that $S$ was isometrically isomorphic to a Banach algebra of Fresnel integrable functions as given by Albeverio and HøeghKrohn [1]. The initial motivation for this paper was a need in [10 and 28] for a Fubini-type stochastic integration formula involving a mix of a one-parameter Wiener process and a multiparameter Wiener process.

For $Q=[O, T]^{N}, N=1,2, \ldots$, let $W=\{W(t): t \in Q\}$ be the stai.dard $N$ parameter Wiener process. Let $\left\{\alpha_{k}\right\}$ be a complete orthonormal (CON) set of functions of bounded variation in the sense of Hardy-Krause [3] on $Q$. For $h$ in $L_{2}(Q)$ let

$$
h_{n}(t)=\sum_{k=1}^{n}\left(h, \alpha_{k}\right) \alpha_{k}(t) .
$$

Then the P.W.Z. integral is defined by the formula

$$
\int_{Q} h(t) d^{*} W(t)=\underset{n \rightarrow \infty}{\operatorname{i} . \mathrm{m}} . \int_{Q} h_{n}(t) d W(t)
$$

where "l.i.m." means "limit in the mean" (or limit in $L_{2}$-sense) and $\int_{Q} h_{n}(t) d W(t)$ is the ordinary Riemann-Stieltjes integral.

It is well known that for each $h$ in $L_{2}(Q)$, the P.W.Z. $\int_{Q} h(t) d^{*} W(t)$ exists almost surely and is essentially independent of the CON set $\left\{\alpha_{K}\right\}$. Also if $h$ is of bounded variation on $Q$ then $\int_{Q} h(t) d^{*} W(t)$ equals the R-S integral $\int_{Q} h(t) d W(t)$ almost surely. Some more general P.W.Z. stochastic integrals are considered in Park [25] and Kuo and Russek [22].

For a nonanticipating function $h(t, \omega)$ in $L_{2}(Q \times \Omega)$, where $(\Omega, \mathscr{F}, P)$ is a probability space, Itô's stochastic integral $\int_{Q} h(t, \omega) d W(t)$ is extremely well known and

Received by the editors March 28, 1987. Presented to the Society, January 21, 1987 under the same title.

1980 Mathematics Subject Classification (1985 Revision). Primary 60H05, 60J65.

Research partially supported by NSF Grant \#DMS-8403197 and by UNL Research Council. 
widely used. In $\S 2$ of this paper we establish some conditions under which the Itô stochastic integral and the P.W.Z. stochastic integral agree almost surely. We then proceed to develop a Fubini theorem for stochastic integrals which unifies several integration formulas.

In $\S 3$ we define a P.W.Z. type double stochastic integral and show that it is equal almost surely to certain iterated stochastic integrals. We then establish a Fubinitype theorem which allows the interchange of the order of the stochastic processes and point out that a corresponding result is not possible for Itô integrals.

2. Stochastic integrals with respect to two independent processes. In our first theorem we show that under certain conditions the Itô and P.W.Z. stochastic integrals agree almost surely.

THEOREM 1. Let $W$ and $X$ be two independent $N$-parameter Wiener processes defined on $Q=[O, T]^{N}$. Let $h(t, u)$ be a function on $Q \times \mathbf{R}$ such that $\int_{Q} E|h(t, X(t))|^{2} d t<\infty$. Then

$$
\int_{Q} h(t, X(t)) d^{*} W(t)=\int_{Q} h(t, X(t)) d W(t)
$$

almost surely, where the right-hand side is the Itô stochastic integral.

PROOF. Let $\left\{\alpha_{k}(t)\right\}_{k=1}^{\infty}$ be a CON set on $Q$ with each $\alpha_{k}$ of bounded variation. Then

$$
\begin{aligned}
E\left[\sum_{k=1}^{n} \int_{Q} h(t, X(t)) \alpha_{k}(t) d t \int_{Q} \alpha_{k}(s) d W(s)-\int_{Q} h(t, X(t)) d W(t)\right]^{2} \\
=E\left[\sum_{k=1}^{n}\left\{\int_{Q} h(t, X(t)) \alpha_{k}(t) d t\right\}^{2}\right]+\int_{Q} E|h(t, X(t))|^{2} d t \\
-2 E\left[\sum_{k=1}^{n} \int_{Q} h(t, X(t)) \alpha_{k}(t) d t \int_{Q} \alpha_{k}(s) d W(s) \int_{Q} h(t, X(t)) d W(t)\right] \\
=E\left[\sum_{k=1}^{n}\left\{\int_{Q} h(t, X(t)) \alpha_{k}(t) d t\right\}^{2}\right]+\int_{Q} E|h(t, X(t))|^{2} d t \\
-2 E\left[\sum_{k=1}^{n}\left\{\int_{Q} h(t, X(t)) \alpha_{k}(t) d t\right\}^{2}\right]
\end{aligned}
$$

$\rightarrow 0$ as $n \rightarrow \infty$ by Parseval's equation.

Therefore

$$
\begin{aligned}
\int_{Q} h(t, X(t)) d W(t) & =\underset{n \rightarrow \infty}{\operatorname{i} . \mathrm{m}} \cdot \sum_{k=1}^{n} \int_{Q} h(t, X(t)) \alpha_{k}(t) d t \int_{Q} \alpha_{k}(s) d W(s) \\
& =\int_{Q} h(t, X(t)) d^{*} W(t)
\end{aligned}
$$

almost surely. 
COROLLARY 1.1. Under the same assumptions as in Theorem 1, if $B$ is a Borel subset of $Q$, then

$$
\int_{B} h(t, X(t)) d^{*} W(t)=\int_{B} h(t, X(t)) d W(t)
$$

almost surely.

COROLlaRY 1.2. If $h$ is in $L_{2}(Q)$, then

$$
\int_{Q} h(t) d^{*} W(t)=\int_{Q} h(t) d W(t)
$$

almost surely.

The following stochastic integration by parts formula [13, p. 268] (also stated in $[19$, p. 283] is well known.

THEOREM A. If $W(t)$ and $X(t)$ are two independent standard Wiener processes on $[0, T]$, then for $0 \leq a<b \leq T$,

$$
\int_{a}^{b} X(t) d^{*} W(t)=X(b) W(b)-X(a) W(a)-\int_{a}^{b} W(t) d^{*} X(t)
$$

almost surely.

In [10], Chang, Johnson, and Skoug obtained the following useful stochastic integration formulas.

THEOREM B. If $X(t)$ and $W(s, t)$ are two independent standard Wiener processes on $[0, T]$ and $Q=[0, T]^{2}$, respectively, then

$$
\int_{Q} X(s) d^{*} W(s, t)=\int_{0}^{T} X(s) d^{*} W(s, T)
$$

almost surely. Furthermore, if $h(s, t)$ is of bounded variation on $Q$, then

$$
\int_{Q} h(s, t) X(s) d^{*} W(s, t)=\int_{0}^{T}\left[\int_{B(s)} h(u, v) d W(u, v)\right] d^{*} X(s)
$$

almost surely where $B(s) \equiv[s, T] \times[0, T]$.

Our next result is a Fubini-type theorem which subsumes both theorems $A$ and $B$. In particular note that we simply require the function $g$ to be in $L_{2}$ rather than to be of bounded variation.

THEOREM 2. Let $W(t)$ be a standard Wiener process on $Q=[0, T]^{N}$ and let $Y(s)$ be a standard Wiener process defined on $Q^{\prime}=[0, T]^{k}$. Assume that $W(t)$ and $Y(s)$ are independent. Then for any function $g$ in $L_{2}\left(Q \times Q^{\prime}\right)$, we have that

$$
\int_{Q}\left[\int_{Q^{\prime}} g(s, t) d Y(s)\right] d W(t)=\int_{Q^{\prime}}\left[\int_{Q} g(s, t) d W(t)\right] d Y(s)
$$

almost surely.

Proof. It suffices to show that

$$
E\left\{\int_{Q}\left[\int_{Q^{\prime}} g(s, t) d Y(s)\right] d W(t)-\int_{Q^{\prime}}\left[\int_{Q} g(s, t) d W(t)\right] d Y(s)\right\}^{2}=0 .
$$


Now,

$$
\begin{gathered}
E\left\{\int_{Q}\left[\int_{Q^{\prime}} g(s, t) d Y(s)\right] d W(t)\right\}^{2}=E\left\{\int_{Q}\left[\int_{Q^{\prime}} g(s, t) d Y(s)\right]^{2} d t\right\} \\
=\int_{Q}\left[\int_{Q^{\prime}} g^{2}(s, t) d s\right] d t=\int_{Q^{\prime}}\left[\int_{Q} g^{2}(s, t) d t\right] d s \\
=E\left\{\int_{Q^{\prime}}\left[\int_{Q} g(s, t) d W(t)\right] d Y(s)\right\}^{2} .
\end{gathered}
$$

Also,

$$
\begin{aligned}
E & \left\{\int_{Q}\left[\int_{Q^{\prime}} g(s, t) d Y(s)\right] d W(t) \cdot \int_{Q^{\prime}}\left[\int_{Q} g(s, t) d W(t)\right] d Y(s)\right\} \\
& =E\left\{\int_{Q}\left[\int_{Q^{\prime}} g(s, t) d Y(s)\right] \cdot \int_{Q^{\prime}}\left[\int_{Q} g(s, u) d W(u)\right] d Y(s) d W(t)\right\} \\
& =E\left\{\int_{Q}\left(\int_{Q^{\prime}} g(s, t)\left[\int_{Q} g(s, u) d W(u)\right] d s\right) d W(t)\right\} \\
& =\int_{Q^{\prime}}\left[\int_{Q} g^{2}(s, t) d t\right] d s .
\end{aligned}
$$

Thus, (2.1) is established.

The following corollaries and remarks show that Theorem 2 is indeed quite a general, powerful, and unifying theorem.

COROllary 2.1. Let $W(t)$ and $Y(s)$ be as in Theorem 2 with $k \leq N$. For $t=\left(t_{1}, \ldots, t_{N}\right)$ in $Q$ let $t^{(k)}=\left(t_{1}, \ldots, t_{k}\right)$. Then for any function $h \in \bar{L}_{2}(Q)$, we have

$$
\int_{Q} h(t) Y\left(t^{(k)}\right) d W(t)=\int_{Q^{\prime}}\left[\int_{B_{k}(s)} h(t) d W(t)\right] d Y(s)
$$

almost surely, where $B_{k}(s)=\prod_{j=1}^{k}\left[s_{j}, T\right] \times[0, T]^{N-k}$.

PROOF. Define a function $\xi$ by

$$
\xi\left(s ; t^{(k)}\right)= \begin{cases}1 & \text { if } 0 \leq s_{j} \leq t_{j} \leq T \text { for } j=1, \ldots, k \\ 0 & \text { otherwise }\end{cases}
$$

Then

$$
h(t) Y\left(t^{(k)}\right)=h(t) \int_{0}^{t_{k}} \cdots \int_{0}^{t_{1}} d Y(s)=\int_{Q^{\prime}} h(t) \xi\left(s ; t^{(k)}\right) d Y(s) .
$$

The result now follows by setting $g(s, t)=h(t) \xi\left(s ; t^{(k)}\right)$ in Theorem 2 above.

COROllary 2.2. Let $W(t), Y(s)$ and $h$ be as in Corollary 2.1. Then

$$
\int_{Q} h(t) Y\left(t_{j_{1}}, \ldots, t_{j_{k}}\right) d W(t)=\int_{Q^{\prime}}\left[\int_{B_{k}^{*}(s)} h(t) d W(t)\right] d Y\left(s_{j_{1}}, \ldots, s_{j_{k}}\right)
$$

almost surely where $j_{1}, \ldots, j_{k}$ are $k$ numbers in increasing order chosen from $\{1,2, \ldots, N\}$ and $B_{k}^{*}(s)$ is obtained from $[0, T]^{N}$ by replacing all the $j_{i}$ th factors by $\left[s_{j_{i}}, T\right]$ for $i=1, \ldots, k$. 
REMARK 2.1. It is easy to observe that Corollary 2.1 with $N=k=1$ implies Theorem A above.

REMARK 2.2. It is also easy to observe the Corollary 2.1 with $N=2$ and $k=1$ implies Theorem B. Note that in Theorem $\mathrm{B}, h$ is required to be of bounded variation on $Q$, while in Corollary $2.1, h$ is only required to be in $L_{2}(Q)$.

REMARK 2.3. In [28, Theorem 2.1] we stated a special case of Corollary 2.2 which played a key role in the proof of the main result in [28].

REMARK 2.4. Theorem 2 also implies a stochastic integration (P.W.Z. and Itô) by parts formula where the integrator and integrand are independent standard Wiener processes on $Q=[0, T]^{N}$ for $N=1$ (Theorem A above), 2,3, . We will give the proof for the case $N=2$; the general case is similar but notationally more complicated. The stochastic integration by parts formula is the same as the ordinary Riemann-Stieltjes integration by parts formula [33, p. 415] but with each R-S integral $\int f d g$ replaced with the corresponding P.W.Z. integral $\int f d^{*} g$.

Let $R=[a, b] \times[\alpha, \beta] \subseteq Q=[0, T]^{2}$. Then, using Corollary 2.1, it follows that

$$
\begin{aligned}
\int_{a}^{b} & \int_{\alpha}^{\beta} Y\left(t_{1}, t_{2}\right) d^{*} W\left(t_{1}, t_{2}\right)=\int_{0}^{T} \int_{0}^{T} \chi_{R}\left(t_{1}, t_{2}\right) Y\left(t_{1}, t_{2}\right) d^{*} W\left(t_{1}, t_{2}\right) \\
= & \int_{0}^{T} \int_{0}^{T}\left[\int_{s_{1}}^{T} \int_{s_{2}}^{T} \chi_{R}\left(t_{1}, t_{2}\right) d W\left(t_{1}, t_{2}\right)\right] d^{*} Y\left(s_{1}, s_{2}\right) \\
= & \int_{0}^{a} \int_{0}^{\alpha}\left[\int_{a}^{b} \int_{\alpha}^{\beta} d W\left(t_{1}, t_{2}\right)\right] d^{*} Y\left(s_{1}, s_{2}\right) \\
& +\int_{a}^{b} \int_{0}^{\alpha}\left[\int_{s_{1}}^{b} \int_{\alpha}^{\beta} d W\left(t_{1}, t_{2}\right)\right] d^{*} Y\left(s_{1}, s_{2}\right) \\
& +\int_{0}^{a} \int_{\alpha}^{\beta}\left[\int_{a}^{b} \int_{s_{2}}^{\beta} d W\left(t_{1}, t_{2}\right)\right] d^{*} Y\left(s_{1}, s_{2}\right) \\
& +\int_{a}^{b} \int_{\alpha}^{\beta}\left[\int_{s_{1}}^{b} \int_{s_{2}}^{\beta} d W\left(t_{1}, t_{2}\right)\right] d^{*} Y\left(s_{1}, s_{2}\right)
\end{aligned}
$$

almost surely. We next evaluate the inner integrals above, reduce the resulting two dimensional integrals whenever possible using the corollaries of Theorem 2, simplify, and obtain the stochastic parts formula

$$
\begin{aligned}
\int_{a}^{b} & \int_{\alpha}^{\beta} Y\left(t_{1}, t_{2}\right) d^{*} W\left(t_{1}, t_{2}\right) \\
= & W(b, \beta) Y(b, \beta)-W(b, \alpha) Y(b, \alpha)-W(a, \beta) Y(a, \beta)+W(a, \alpha) Y(a, \alpha) \\
& -\int_{a}^{b}\left[W\left(s_{1}, \beta\right) d^{*} Y\left(s_{1}, \beta\right)-W\left(s_{1}, \alpha\right) d^{*} Y\left(s_{1}, \alpha\right)\right] \\
& -\int_{\alpha}^{\beta}\left[W\left(b, s_{2}\right) d^{*} Y\left(b, s_{2}\right)-W\left(a, s_{2}\right) d^{*} Y\left(a, s_{2}\right)\right] \\
& +\int_{a}^{b} \int_{\alpha}^{\beta} W\left(s_{1}, s_{2}\right) d^{*} Y\left(s_{1}, s_{2}\right)
\end{aligned}
$$

almost surely. 
REMARK 2.5. The stochastic integration by parts formula for $N=2$ was also established in [21] using a very different method.

3. Double stochastic integrals and centered double stochastic integrals. In $\S 2$ we established various Fubini-type theorems for stochastic integrals involving independent standard Wiener processes $Y$ and $W$. In this section we consider the case where $Y=W$. Note that in this case, Theorem 1 no longer holds. For example, in the one-parameter case the P.W.Z. integral $\int_{0}^{T} W(t) d^{*} W(t)$ equals $W^{2}(T) / 2$ almost surely, while the Itô integral $\int_{0}^{T} W(t) d W(t)$ equals $\left(W^{2}(T)-T\right) / 2$ almost surely.

Let $\left\{\alpha_{i}(t)\right\}_{i=1}^{\infty}$ be a CON set on $Q=[0, T]^{N}$ with each $\alpha_{i}$ of bounded variation on $Q$. For $g$ in $L_{2}\left(Q^{2}\right)$, we define the centered double stochastic integral of P.W.Z. by

$$
\begin{aligned}
\left(\text { c) } \int_{Q^{2}} g(s, t) d^{*} W(s) d^{*} W(t)\right. & \\
=\text { l.j.m. }_{n \rightarrow \infty}\left\{\left[\sum_{j=1}^{n} \int_{Q}\left[\sum_{i=1}^{n} \int_{Q} g(s, t) \alpha_{i}(s) d s \cdot \int_{Q} \alpha_{i}(u) d W(u)\right] \alpha_{j}(t) d t\right.\right. & \left.\cdot \int_{Q} \alpha_{j}(v) d W(v)\right] \\
& \left.-\sum_{i=1}^{n} \int_{Q^{2}} g(s, t) \alpha_{i}(s) \alpha_{i}(t) d s d t\right\} .
\end{aligned}
$$

We may rewrite the above as

$$
\begin{aligned}
\text { (c) } \int_{Q^{2}} g(s, t) d^{*} W(s) d^{*} W(t) & \\
= & \text { l.i.m. } \\
n \rightarrow \infty & {\left[\sum_{i, j=1}^{n} \int_{Q^{2}} g(s, t) \alpha_{i}(s) \alpha_{j}(t) d s d t \cdot \int_{Q} \alpha_{i} d W \cdot \int_{Q} \alpha_{j} d W\right.} \\
& \left.\quad-\sum_{i=1}^{n} \int_{Q^{2}} g(s, t) \alpha_{i}(s) \alpha_{i}(t) d s d t\right] .
\end{aligned}
$$

Although defined quite differently, the above centered double stochastic integral behaves very much like the one defined by Shepp [31, p. 333]. Since

$$
E\left[\int_{Q} \alpha_{i} d W \cdot \int_{0} \alpha_{j} d W\right]=\delta_{i j}
$$

we have that

$$
E\left[(\mathrm{c}) \int_{Q^{2}} g(s, t) d^{*} W(s) d^{*} W(t)\right]=0 .
$$

Using the well-known formula (see [30, Problem 4E, p. 95]),

$$
E\left[\int_{Q} \alpha_{i} d W \cdot \int_{Q} \alpha_{j} d W \cdot \int_{Q} \alpha_{p} d W \cdot \int_{Q} \alpha_{q} d W\right]=\delta_{i j} \cdot \delta_{p q}+\delta_{i p} \cdot \delta_{j q}+\delta_{i q} \cdot \delta_{j p}
$$


we have that

$$
\begin{aligned}
& E\left[\sum_{i, j=1}^{n} \int_{Q^{2}} g(s, t) \alpha_{i}(s) \alpha_{j}(t) d s d t \cdot \int_{Q} \alpha_{i} d W\right. \\
& \left.\cdot \int_{Q} \alpha_{j} d W-\sum_{i=1}^{n} \int_{Q^{2}} g(s, t) \alpha_{i}(s) \alpha_{i}(t) d s d t\right]^{2} \\
& =\sum_{i, j=1}^{n}\left[\int_{Q^{2}} g(s, t) \alpha_{i}(s) \alpha_{j}(t) d s d t\right]^{2} \\
& \quad+\sum_{i, j=1}^{n}\left[\int_{Q^{2}} g(s, t) \alpha_{i}(s) \alpha_{j}(t) d s d t\right]\left[\int_{Q^{2}} g(s, t) \alpha_{j}(s) \alpha_{i}(t) d s d t\right]
\end{aligned}
$$

Due to Parseval's equation, we obtain

$$
E\left[(\mathrm{c}) \int_{Q^{2}} g(s, t) d^{*} W(s) d^{*} W(t)\right]^{2}=\int_{Q^{2}} g^{2}(s, t) d s d t+\int_{Q^{2}} g(s, t) g(t, s) d s d t
$$

As is well known (see [16]), Itô's double stochastic integral $\int_{Q^{2}} g(s, t) d W(s) d W(t)$ has expected value 0 and variance $2 \int_{Q^{2}} g^{2}(s, t) d s d t$. So,

$$
\text { (c) } \int_{Q^{2}} g(s, t) d^{*} W(s) d^{*} W(t)
$$

and $\int_{Q^{2}} g(s, t) d W(s) d W(t)$ have different variances unless $g(s, t)$ is symmetric in $s$ and $t$.

Let $\mathscr{A}$ consist of all functions $g \in L_{2}\left(Q^{2}\right)$ such that

$$
\sum_{i=1}^{\infty} \int_{Q^{2}} g(s, t) \alpha_{i}(s) \alpha_{i}(t) d s d t
$$

converges for some CON set $\left\{\alpha_{i}(t)\right\}$ on $Q$ with each $\alpha_{i}(t)$ of bounded variation. Park [27] shows that for a large class of functions $g \in L_{2}\left(Q^{2}\right)$,

$$
\sum_{i=1}^{\infty} \int_{Q^{2}} g(s, t) \alpha_{i}(s) \alpha_{i}(t) d s d t=\int_{Q} g(s, s) d s
$$

In particular, this class contains all the continuous functions on $Q^{2}$.

Let $g \in \mathscr{A}$. Define the double stochastic integral of P.W.Z. by

$$
\begin{array}{rl}
\int_{Q^{2}} & g(s, t) d^{*} W(s) d^{*} W(t) \\
& =\underset{n \rightarrow \infty}{\lim . \operatorname{m}} \sum_{i, j=1}^{n} \int_{Q^{2}} g(s, t) \alpha_{i}(s) \alpha_{j}(t) d s d t \cdot \int_{Q} \alpha_{i} d W \cdot \int_{Q} \alpha_{j} d W .
\end{array}
$$

It follows that

$$
\begin{aligned}
\int_{Q^{2}} g(s, t) d^{*} W(s) d^{*} W(t)= & (\mathrm{c}) \int_{Q^{2}} g(s, t) d^{*} W(s) d^{*} W(t) \\
& +\sum_{i=1}^{\infty} \int_{Q^{2}} g(s, t) \alpha_{i}(s) \alpha_{i}(t) d s d t
\end{aligned}
$$


Therefore

$$
E\left[\int_{Q^{2}} g(s, t) d^{*} W(s) d^{*} W(t)\right]=\sum_{i=1}^{\infty} \int_{Q^{2}} g(s, t) \alpha_{i}(s) \alpha_{i}(t) d s d t
$$

and

$$
\operatorname{Var}\left[\int_{Q^{2}} g(s, t) d^{*} W(s) d^{*} W(t)\right]=\int_{Q^{2}} g^{2}(s, t) d s d t+\int_{Q^{2}} g(s, t) g(t, s) d s d t .
$$

For $g \in \mathscr{A}$ define the iterated stochastic integral by the formula

$$
\int_{Q}\left[\int_{Q} g(s, t) d W(s)\right] d^{*} W(t)=\underset{n \rightarrow \infty}{\operatorname{ij} . \mathrm{m}} \sum_{i=1}^{n} \int_{Q}\left[\int_{Q} g(s, t) d W(s)\right] \alpha_{i}(t) d t \cdot \int_{Q} \alpha_{i} d W .
$$

We have the following.

THEOREM 3. If $g \in \mathscr{A}$, then, almost surely,

$$
\int_{Q^{2}} g(s, t) d^{*} W(s) d^{*} W(t)=\int_{Q}\left[\int_{Q} g(s, t) d W(s)\right] d^{*} W(t) .
$$

PROOF. The theorem follows by observing that

$$
\begin{aligned}
E\left\{\sum_{i, j=1}^{n} \int_{Q^{2}} g(s, t) \alpha_{i}(s) \alpha_{j}(t) d s d t \cdot \int_{Q} \alpha_{i} d W \cdot \int_{Q} \alpha_{j} d W\right. \\
\left.-\sum_{k=1}^{m} \int_{Q}\left[\int_{Q} g(s, t) d W(s)\right] \alpha_{k}(t) d t \cdot \int_{Q} \alpha_{k} d W\right\}^{2} \rightarrow 0
\end{aligned}
$$

as $m, n \rightarrow \infty$.

COROllary 3.1. If $g \in \mathscr{A}$, then, almost surely,

$$
\int_{Q}\left[\int_{Q} g(s, t) d W(s)\right] d^{*} W(t)=\int_{Q}\left[\int_{Q} g(s, t) d W(t)\right] d^{*} W(s) .
$$

PrOOF. By the definition,

$$
\int_{Q^{2}} g(s, t) d^{*} W(s) d^{*} W(t)=\int_{Q^{2}} g(s, t) d^{*} W(t) d^{*} W(s) .
$$

Therefore, the result follows from Theorem 3.

THEOREM 4. Let $h \in L_{2}(Q)$. For $t=\left(t_{1}, \ldots, t_{N}\right) \in Q$ and $k=1,2, \ldots, N$, let $t^{(k)}=\left(t_{1}, \ldots, t_{k}\right), t_{T}^{(k)}=\left(t_{1}, \ldots, t_{k}, T, \ldots, T\right)$ and $\tilde{t}^{(k)}=\left(t_{k+1}, \ldots, t_{N}\right)$. Then for $k=1,2, \ldots, N$,

$$
\int_{Q} h(t) W\left(t_{T}^{(k)}\right) d^{*} W(t)=\int_{Q}\left[\int_{B_{k}(s)} h(t) d W(t)\right] d^{*} W(s)
$$

almost surely where $B_{k}(s)=\prod_{j=1}^{k}\left[s_{j}, T\right] \times[0, T]^{N-k}$.

Proof. Using the function $\xi$ given by (2.2), we may write

$$
h(t) W\left(t_{T}^{(k)}\right)=\int_{Q} h(t) \xi\left(s^{(k)} ; t^{(k)}\right) d W(s)
$$


almost surely. Then using Corollary 3.1 we obtain

$$
\begin{aligned}
\int_{Q} h(t) W\left(t_{T}^{(k)}\right) d^{*} W(t) & =\int_{Q}\left[\int_{Q} h(t) \xi\left(s^{(k)} ; t^{(k)}\right) d W(s)\right] d^{*} W(t) \\
& =\int_{Q}\left[\int_{Q} h(t) \xi\left(s^{(k)} ; t^{(k)}\right) d W(t)\right] d^{*} W(s) .
\end{aligned}
$$

Thus, the result follows from Theorem 3 , provided the function $h(t) \xi\left(s^{(k)} ; t^{(k)}\right) \in$ $\mathscr{A}$. Now, choose a CON set $\left\{\beta_{p}\left(s^{(k)}\right)\right\}_{p=1}^{\infty}$ on $[0, T]^{k}$ satisfying:

$$
\underset{n \rightarrow \infty}{\lim .} \sum_{i=1}^{n} \beta_{p}\left(t^{(k)}\right) \int_{0}^{t_{k}} \cdots \int_{0}^{t_{1}} \beta_{p}\left(s^{(k)}\right) d s_{1} \cdots d s_{k}=2^{-k} \text {. }
$$

For the existence of such a CON set, see Park [25, pp. 391-92]. Also, choose a CON set $\left\{\gamma_{q}\left(\tilde{s}^{(k)}\right)\right\}_{q=1}^{\infty}$ on $[0, T]^{N-k}$, and set $\left\{\alpha_{i}(s)\right\}=\left\{\beta_{p}\left(s^{(k)}\right) \gamma_{q}\left(\tilde{s}^{(k)}\right)\right\}$. Then,

$$
\begin{aligned}
& \sum_{i=1}^{\infty} \int_{Q^{2}} h(t) \xi\left(s^{(k)} ; t^{(k)}\right) \alpha_{i}(s) \alpha_{i}(t) d s d t \\
&=\sum_{p, q=1}^{\infty} \int_{Q} h(t)\left[\beta_{p}\left(t^{(k)}\right) \int_{0}^{t_{k}} \cdots\right.\left.\int_{0}^{t_{1}} \beta_{p}\left(s^{(k)}\right) d s_{1} \cdots d s_{k}\right] \\
& \cdot\left[\gamma_{q}\left(\tilde{t}^{(k)}\right) \int_{[0, T]^{N-k}} \gamma_{q}\left(\tilde{s}^{(k)}\right) d \tilde{s}^{(k)}\right] d t \\
&=2^{-k} \int_{Q} h(t) d t .
\end{aligned}
$$

Therefore, $h(t) \xi\left(s^{(k)} ; t^{(k)}\right) \in \mathscr{A}$.

Our final corollary is a Fubini-type theorem similar to Corollary 2.1.

COROLlaRY 4.1. Let $h \in L_{2}(Q)$. Then, for $k=1,2, \ldots, N$,

$$
\int_{Q} h(t) W\left(t_{T}^{(k)}\right) d^{*} W(t)=\int_{[0, T]^{k}}\left[\int_{B_{k}(s)} h(t) d W(t)\right] d^{*} W\left(s_{T}^{(k)}\right)
$$

almost surely, where the right-hand side is a $P$.W.Z. stochastic integral with respect to a CON set on $[0, T]^{k}$.

ProOF. Because of Theorem 4, it suffices to show that

$$
\int_{Q}\left[\int_{B_{k}(s)} h(t) d W(t)\right] d^{*} W(s)=\int_{[0, T]^{k}}\left[\int_{B_{k}(s)} h(t) d W(t)\right] d^{*} W\left(s_{T}^{(k)}\right)
$$

almost surely. However, this follows almost immediately by observing that

$$
\int_{[0, T]^{k}} \alpha\left(s^{(k)}\right) d W\left(s_{T}^{(k)}\right)=\int_{Q} \alpha\left(s^{(k)}\right) d W(s) .
$$

REMARK. One cannot establish a theorem like Theorem 4 in terms of Itô's stochastic integral, because $W\left(t_{T}^{(k)}\right)$ is not measurable with respect to the $\sigma$-field generated by $\left\{W(s): s_{i} \leq t_{i}, 1 \leq i \leq N\right\}$. Thus, the left-hand side of (3.1) is not defined in the sense of Itô. 


\section{REFERENCES}

1. S. Albeverio and R. Høegh-Krohn, Mathematical theory of Feynman path integrals, Lecture Notes in Math., vol. 523, Springer-Verlag, 1976.

2. L. Arnold, Stochastic differential equations: theory and applications, Wiley, 1974.

3. E. Berkson and T. A. Gillespie, Absolutely continuous functions of two variables and well-bounded operators, J. London Math. Soc. 30 (1984), 305-321.

4. R. H. Cameron and D. A. Storvick, Some Banach algebras of analytic Feynman integrable functionals, Analytic Functions, Kozubnik, 1979, Lecture Notes in Math., vol. 798, SpringerVerlag, 1980, pp. 18-67.

5. __ A new translation theorem for the analytic Feynman integral, Rev. Roumaine Math. Pure Appl. 27 (1982), 937-944.

6. $\ldots$ A simple definition of the Feynman integral, with applications, Mem. Amer. Math. Soc. 46 (1983), 1-46.

7. London Math. Soc. 52 (1986), 557-581.

8. __, Change of scale formulas for Wiener integrals, Suppl. Rend. Circ. Mat. Palermo, Proc. Conf. on Functional Integration held in Sherbrooke, Canada, July, 1986 (to appear).

9. __ Relationships between the Wiener integral and the analytic Feynman integral, Suppl. Rendi. Circ. Palermo, Proc. Conf. on Functional Integration held in Sherbrooke, Canada, July, 1986 (to appear).

10. K. S. Chang, G. W. Johnson, and D. L. Skoug, The Feynman integral of quadratic potentials depending on two time variables, Pacific J. Math. 122 (1986), 11-33.

11. _ Necessary and sufficient conditions for membership in the Banach algebra $S$ for certain classes of functions, Suppl. Rend. Circ. Mat. Palermo, Proc. Conf. on Functional Integration held in Sherbrooke, Canada, July, 1986 (to appear).

12. __ functions in the Banach algebra $S(\nu)$, J. Korean Math. Soc. 24 (1987).

13. I. I. Gihman and A. V. Skorohod, The theory of stochastic processes. III, Springer-Verlag, 1979.

14. B. Hajek and E. Wong, Set-parametered martingales and multiple stochastic integration (Proc. LMS Durham Symp.), Lecture Notes in Math., vol. 851, Springer-Verlag, 1980, pp. 119-151.

15. K. Itô, Stochastic integral, Proc. Imperial Acad. Tokyo 20 (1944), 519-524.

16. __ Multiple Wiener integral, J. Math. Soc. Japan 3 (1951), 157-169.

17. G. W. Johnson, The equivalence of two approaches to the Feynman integral, J. Math. Phys. 23 (1982), 2090-2096.

18. G. W. Johnson and D. L. Skoug, Notes on the Feynman integral. I, Pacific J. Math. 93 (1981), 313-324.

19. __ Notes on the Feynman integral. II, J. Functional Analysis 41 (1981), 277-289.

20. _ Notes on the Feynman integral. III, The Schroedinger equation, Pacific J. Math. 105 (1983), 321-358.

21. $\_$, A stochastic integration formula for two-parameter $\times$two-parameter Wiener space, Siam J. Math Anal. 18 (1987), 919-932.

22. H. Kuo and A. Russek, White noise approach to stochastic integration, J. Multivariate Analysis (to appear).

23. H. P. McKean, Stochastic integrals, Academic Press, New York, 1969.

24. R. E. A. C. Paley, N. Wiener and A. Zygmund, Notes on random functions, Math. Z. 37 (1933), 647-688.

25. C. Park, A generalized Paley-Wiener-Zygmund integral and its applications, Proc. Amer. Math. Soc. 23 (1969), 388-400.

26. __ On Fredholm transformations in Yeh-Wiener space, Pacific J. Math. 40 (1972), 173-195.

27. $\ldots$, An extension of Parseval's equation, SIAM J. Math. Anal. 6 (1975), 714-723.

28. C. Park and D. L. Skoug, The Feynman integral of quadratic potentials depending on $n$ time variables Nagoya Math. J. (to appear).

29. W. J. Park, A multiparameter Gaussian process, Ann. Math. Statist. 41 (1970), 1582-1595.

30. E. Parzen, Stochastic processes, Holden-Day, San Francisco, Calif., 1967. 
31. L. A. Sheep, Radon-Nikodym derivative of Gaussian measures, Ann. Math. Statist. 37 (1966), 321-354.

32. E. Wong and M. Zakai, Martingales and stochastic integrals for processes with a multi-dimensional parameter, Z. Wahrsch. Verw. Gebiete 29 (1974), 109-122.

33. J. Yeh, Cameron-Martin translation theorems in the Wiener space of functions of two variables, Trans. Amer. Math. Soc. 107 (1963), 409-420.

Department of Mathematics and Statistics, Miami University, OXford, Ohio 45056

Department of Mathematics and Statistics, University of Nebraska, LinCOLN, NEBRASKA 68588-0323 